\title{
Algunas cuestiones sobre política educativa y la enseñanza de lenguas extranjeras durante el franquismo*
}

\author{
María Jesús Vera-Cazorla
}

\begin{abstract}
Afiliada institucionalmente a la Universidad de Las Palmas de Gran Canaria (España). Correo electrónico: mariajesus.vera@ulpgc.es. La autora es doctora en Filología Moderna de la Universidad de Las Palmas de Gran Canaria (España). Entre sus publicaciones recientes tenemos: "La evaluación entre iguales: estudio de caso en la Universidad de Las Palmas de Gran Canaria”, Revista Innovación Educativa No. 27 (2017) y "Tradition and innovation in foreign language teaching in Gran Canaria in the nineteenth century", History of Education and Children's Literature Vol. XII No. 2 (2017). Entre sus temas de interés están Historia de la enseñanza de lenguas extranjeras, Métodos innovadores para evaluación en la universidad, y Lengua y cultura.
\end{abstract}

\footnotetext{
Recibido: 5 de abril de 2017

Aprobado: 10 de septiembre de 2017

Modificado: 23 de septiembre de 2017

Artículo de investigación científica
}

DOI: http://dx.doi.org/10.15648/hc.33.2018.3

\footnotetext{
* $\quad$ Este artículo forma parte del proyecto: "Cuestiones sobre política educativa y la enseñanza de lenguas extranjeras durante el franquismo" financiación propia. Esta publicación está bajo una licencia Creative Commons Reconocimiento-NoComercial 4.0
} 
Algunas cuestiones sobre política educativa y la enseñanza de lenguas extranjeras durante el franquismo

\title{
Resumen
}

En este trabajo se analiza la presencia de las lenguas extranjeras en el currículo académico tanto en la enseñanza primaria como en la formación de maestros durante el franquismo. Especialmente en la última etapa del franquismo, se observa un cambio en la percepción de la importancia del estudio de las lenguas extranjeras y la necesidad de incorporar dicha formación en los distintos niveles educativos en una España que empieza a abrirse a Europa y en la que el sector turismo adquiere cada vez más importancia; de ahí la inclusión de la especialidad de Lengua en la formación inicial del Magisterio.

Palabras clave: enseñanza de lenguas extranjeras, formación de maestros.

\section{Some issues about educational policy and the teaching of foreign languages during the Francoism}

\begin{abstract}
This paper analyzes the presence of foreign languages in the academic syllabus in the context of primary education and the training of teachers during the Franco's regime. Especially in the last period of Francoism, it is observed a change concerning the perception of the importance of the study of foreign languages and the need to incorporate it in the different levels of education in Spain as the country began to open to Europe and at a time when tourism was becoming increasingly important; hence the inclusion of foreign languages in the early training of the Faculty.
\end{abstract}

Key words: foreign language teaching, teachers' training.

Algumas questões sobre política educativa e o ensino de línguas estrangeiras durante o franquismo

\section{Resumo}

Neste trabalho analisa-se a presença das línguas estrangeiras no currículo académico tanto no ensino primário como na formação de maestros durante o franquismo. Especialmente na última etapa do franquismo, observa-se uma mudança na percepção da importância do estudo das línguas estrangeiras e a necessidade de incorporar dita 
formação nos diferentes níveis educativos numa Espanha que começa a se abrir a Europa e na que o setor turismo adquire a cada vez mais importância; daí a inclusão da especialidad de Língua na formação inicial do Magisterio.

Palavras-chave: ensino de línguas estrangeiras, formação de Professores.

Quelques questions de politique éducative et l'enseignement des langues étrangères durant le franquisme

\section{Résumé}

Ce travail analyse la présence des langues étrangères dans le programme académique tant dans l'enseignement primaire que dans la formation d'enseignants durant le franquisme. Tout particulièrement, dans la dernière étape du franquisme, on observe un changement dans la perception de l'importance de l'étude des langues étrangères et le besoin d'incorporer cette formation dans les différents niveaux éducatifs d'une Espagne qui commence à s'ouvrir à l'Europe et dans laquelle le secteur tourisme acquiert de plus en plus d'importance ; d'où l'inclusion de la spécialité Langue dans la formation initiale du corps enseignant.

Mots clés: enseignement des langues étrangères, formation d'enseignants.

\section{INTRODUCCIÓN}

Cada año la Comisión Europea presenta una evaluación anual de los sistemas de educación y formación en toda Europa. El informe reúne los últimos datos cuantitativos y cualitativos sobre las medidas adoptadas en materia educativa por los diferentes estados miembros de la Unión Europea. El objetivo es contribuir a la implementación del marco de cooperación sobre educación y formación ET 2020, por lo que esta memoria es una herramienta para que las autoridades educativas comparen su país con otros estados miembros de la UE.

En el informe del año 2016 la comisión vuelve a incidir sobre lo importante que es para Europa que sus ciudadanos hablen varias lenguas comunitarias. Para conseguir mejores oportunidades para la vida y el trabajo en la economía y la sociedad de hoy tras el aumento de la globalización y la 
migración dentro y hacia dentro de la UE, la habilidad de comunicarse en la propia lengua materna y en las lenguas extranjeras ocupa un lugar prominente entre las competencias clave requeridas:

"La construcción de Europa como espacio de libre circulación de personas y un mercado único supone integrar su diversidad lingüística. Además de sus 24 lenguas oficiales, la UE alberga alrededor de 60 lenguas indígenas regionales y una riqueza adicional de lenguas no europeas traídas por inmigrantes recién llegados y personas de origen inmigrante. Ser capaz de comunicarse a través de esta diversidad es fundamental para el progreso económico, la cohesión social y el diálogo intercultural"1.

En las conclusiones del Consejo Europeo de Barcelona de marzo de 2002, la UE reconoce la importancia de dominar las lenguas extranjeras y se compromete a mejorar su competencia mediante la enseñanza de al menos dos lenguas extranjeras desde una edad muy temprana. En 2014, la UE estaba aún lejos de alcanzar este objetivo, ya que solo el $60 \%$ de los alumnos aprendían dos o más lenguas extranjeras en los primeros niveles de enseñanza secundaria. Sin embargo, a pesar de que la primera lengua extranjera se imparte desde la enseñanza primaria en la mayoría de los estados miembros y de que 3 de cada 4 estudiantes aprenden al menos dos lenguas extranjeras en los primeros niveles de enseñanza secundaria en más de la mitad de los estados miembros de la UE, los datos disponibles muestran que la competencia en idiomas extranjeros sigue siendo insatisfactoria. En cuanto a la población adulta, la encuesta de educación de adultos reveló que en 2011 el 34 \% de los europeos afirmaba que no sabía ninguna lengua extranjera, $36 \%$ sabía un idioma, el $21 \%$ afirmó saber más de un idioma extranjero y menos del $10 \%$ de los encuestados afirmaron tener conocimiento de tres o más lenguas extranjeras ${ }^{2}$.

Uno de los objetivos de la política de multilingüismo de la UE es que to-

241 Traducción propia. European Comission. Education and Training Monitor 2016. https://ec.europa. eu/education/sites/education/files/monitor2016_en.pdf (consulta 21 de diciembre de 2016).

2 European Comission. Education and Training Monitor 2016. https://ec.europa.eu/education/sites/education/files/monitor2016_en.pdf (consulta 21 de diciembre de 2016). 
dos los europeos hablen dos idiomas además de su lengua materna. Para ello es importante que los niños entren desde edad temprana en contacto con dos lenguas extranjeras, pues se ha demostrado que esto no solo acelera el aprendizaje de los idiomas, sino que además mejora las competencias en la lengua materna ${ }^{3}$.

Sin embargo, esta apuesta por el aprendizaje de lenguas extranjeras dista mucho de lo que ocurría en España en la época franquista. Debido a la ruina económica y al bloqueo internacional tras la Guerra Civil, el estudio de las lenguas modernas no figuraba entre las prioridades de Franco para la reconstrucción del país. El nuevo régimen nacionalista no parecía proclive a la cultura extranjera, ni a cualquier otra cultura que no fuese la castellana, por lo que entre 1938 y 1940 se promulgó mucha normativa prohibiendo el uso público (en rótulos, muestras, anuncios y similares) de vocablos no castellanos ${ }^{4}$.

Sin embargo, a finales de los años 50 la actitud del gobierno franquista hacia el estudio de las lenguas extranjeras cambia. Anteriormente, la única titulación que incluía lenguas extranjeras era la de Filosofía y Letras, pero en ese momento se crea la sección de Filología Moderna dentro de esa Facultad. Estas enseñanzas de lenguas extranjeras se implantaron con carácter provisional en el curso 1952/53 en la Universidad de Salamanca y en la Universidad de Madrid en el curso siguiente. ${ }^{5}$ La creación de los nuevos estudios coincidió con el inicio de las conversaciones con los Estados Unidos que conducirían al acuerdo militar firmado el 24 de septiembre de 1953, el cual propició la aceptación internacional del Gobierno de Franco y la admisión de España como miembro de las Naciones Unidas en 1955. Los estudios universitarios de lenguas extranjeras no solo aspiraban a poner fin al proverbial abandono de la enseñanza del idioma en el sistema educativo español, sino también eran un signo de que España estaba negociando un nuevo rumbo en su devenir histórico ${ }^{6}$.

3 Disponible en https://europa.eu/european-union/topics/multilingualism_es.

4 Tomás Monterrey, "Los estudios ingleses en España (1900-1950): legislación curricular", Atlantis Vol. 25 Núm. 1 (2003): 73.

5 Carmen Morales Gálvez et al. La enseñanza de lenguas extranjeras en España (Madrid: Secretaría General Técnica. Subdirección General de Información y Publicaciones, 2000), 75-76.

6 Tomás Monterrey, "Los estudios ingleses...”, 65. 
Las lenguas extranjeras no solo empezaron a tener presencia propia en los estudios universitarios en la década de los 50 del siglo XX, sino también en las escuelas de primaria. Madrid ${ }^{7}$ menciona que fue en la Ley de Educación Primaria de 1945 cuando se propuso introducir en la escuela la enseñanza del inglés por vez primera, si bien fue con la Ley General de Educación (LGE) de 1970 cuando los idiomas modernos experimentaron un auge extraordinario. Concretamente, el idioma moderno se empieza a enseñar en el Ciclo Superior de la Educación General Básica (EGB) a partir de $6^{\circ}$, que correspondía a los 12 años de edad. Ese nivel era impartido por los maestros, lo que tuvo importantes repercusiones en la formación de los mismos.

"Como respuesta a esta nueva situación y ante la necesidad de que los maestros fuesen formados en una materia que tendrían que impartir, en 1971 se implantó un nuevo plan de estudios de maestros que recogía la existencia de la especialidad de Lengua Española e idiomas modernos, considerándose obligatorio el estudio de las materias Lengua y Literatura Francesas y Lengua y Literatura Inglesas en todos los cursos. Dicho plan tuvo carácter experimental hasta 1977 en que una Orden Ministerial lo generalizó"s.

En este trabajo se examina la presencia de las lenguas extranjeras en los planes de estudios de las Escuelas Normales ${ }^{9}$ durante el franquismo, es decir, desde 1936 hasta 1975, y en la LGE de 1970. Posteriormente, se estudia la metodología empleada para la enseñanza de lengua inglesa con los alumnos de la segunda etapa de la EGB en las décadas de los 70 y 80 del siglo XX para finalmente analizar la serie de libros de texto más representativa de esta época, Ready, Steady, Go de Carmen Echevarría y José Merino, publicada por la editorial Anaya a principio de la década de los 70 del siglo pasado.

7 Daniel Madrid, "Problemática de la enseñanza de las lenguas extranjeras en España", en La enseñanza de lenguas en el nuevo milenio, eds. R. Gómez-Caminero (Granada: Grupo Editorial Universitario, 2001), 11.

8 Carmen Morales Gálvez et al. La enseñanza de lenguas..., 77.

9 Las instituciones educativas encargadas de la formación de los maestros de escuela primaria. 


\section{La lengua eXtranjera EN LOS PLANES de ESTUdios de las ESCUElas NORMALES DURANTE EL FRANQUISMO (1936-1975)}

El esfuerzo realizado por el gobierno de la República para elevar el nivel socio-profesional del profesorado de enseñanza primaria quedó truncado tras la guerra civil. La censura y el control ideológico pronto marcaron el comportamiento de la sociedad española:

"El aparato escolar republicano fue desplazado hasta su total eliminación y sustituido por una nueva escuela que reivindicaba los valores religiosos y patrios propulsados por la iglesia y la falange [...] En la enseñanza pública el Estado se limitó a la depuración y reclutamiento de los cuadros del magisterio nacional, así como a la impartición de un currículo inspirado en el nacionalcatolicismo"10.

El Plan Bachiller de $1940^{11}$, si se puede llamar así a un curso acelerado que facultaba a los bachilleres para ejercer el Magisterio, no incluía el estudio de ninguna lengua moderna. En 1942 se publica el primer plan de estudios franquista propiamente dicho. A los aspirantes se les exigía tener 12 años cumplidos y aprobar un ejercicio de ingreso que consistía en una redacción, lectura de un texto, resolución de problemas sencillos y algunas preguntas elementales sobre otras materias ${ }^{12}$. Este plan constaba de tres cursos académicos de formación cultural y un año de formación profesional. Durante los tres años de formación cultural, los alumnos aprendían francés -si bien en primer curso no se especifica el idioma, sino que se menciona una lengua moderna. Para Ávila y Holgado, los aspectos relacionados con los componentes didácticos y metodológicos apenas son tratados en este plan. Estos autores subrayan que:

10 Teresa González Pérez, Enseñando a enseñar. Las escuelas de magisterio (Madrid: Mercurio Editorial, 2013), 69-70.

11 Alejandro Ávila Fernández y Juan A. Holgado Barroso, Formación del Magisterio en España. La legislación normalista como instrumento de poder y control (1834-2007) (Madrid: Ministerio de Educación, Política Social y Deporte. Secretaría General de Educación. Subdirección General de Información y Publicaciones, 2008).

12 Teresa González Pérez, Enseñando a enseñar, 79. 
"El rasgo dominante será la finalidad ideológica, lógica consecuencia del papel transcendental concedido a la figura del maestro en el pensamiento nacional-catolicista, cuya formación será doctrinal, monolítica, dogmática, unidimensional, tendenciosa, pobre en contenidos y bajo una identidad cultural cerrada e igualmente pobre, en consonancia con la miseria social y cultural de estos años" 13 .

Con el Plan de 1950 se intenta mejorar el nivel formativo de los aspirantes a maestros exigiéndoles haber cursado el bachillerato elemental. Durante los tres cursos de la carrera solo se imparte un idioma extranjero, generalmente el francés, en el tercer año, si bien

“disciplinas como Música, Francés o Enseñanzas del hogar reciben menor atención y desarrollo e incluso no llegan a relacionarse como temas numerados y ordenados, sino presentados en forma de grandes apartados, con lo cual se concede mayor libertad docente a la hora de impartir el programa. Evidentemente, el interés se centra en aquellas asignaturas con una mayor carga política e ideológica: Historia, Formación del Espíritu Nacional o Educación Física y Deportes"14.

España comienza a salir del ostracismo en la década de los 50, integrándose en la ONU y en la UNESCO. En los años 60 la economía española intenta implantar un nuevo modelo basado en el desarrollo capitalista occidental, lo que exige la transformación profunda del sistema educativo, llamado a convertirse en motor del crecimiento de acuerdo con los principios tecnocráticos. Consecuentemente, se impone un replanteamiento de los contenidos y de la finalidad de la enseñanza. Se entiende que los contenidos deben estar ligados a la aplicación práctica y basarse en la psicología individual y del aprendizaje $\mathrm{e}^{15}$. 
"El perfil del maestro de las décadas anteriores no puede responder a las exigencias del modo de educación tecnocrático de masas, solicitándose un cambio en su formación inicial. El Plan de 1967 responde a este tránsito y plantea los fundamentos para la posterior reforma de 1971 y, en cierto sentido, adelanta también un conjunto de ideas reflejadas con posterioridad en la Ley General de Educación de 1970"16.

Con la Ley de 1967 se produce una nueva reforma de los estudios de Magisterio exigiéndose a los futuros maestros el título de bachiller superior. Con el Plan de Estudios de 1967, que establece una escolaridad de dos cursos, se incrementa el número de materias psicopedagógicas, fortaleciéndose la presencia de las Didácticas especiales al tiempo que se introducía un curso completo de prácticas docentes. La asignatura de Idioma Inglés y su didáctica se impartía en los dos cursos. Se intentaba desarrollar un modelo de enseñanza tecnocrática más adecuado a los cambios tecnológicos y productivos que estaban registrándose en España ${ }^{17}$.

En el Plan de Estudios Experimental de 1971, reformado en 1977, se establece el Curso de Orientación Universitaria (C.O.U.) como requisito para el acceso a los estudios de Magisterio que tendrán una duración de tres años. El rasgo fundamental del plan será la especialización, dada la necesidad de contar con maestros capacitados para impartir docencia en la EGB. La especialidad de Filología incluye las asignaturas de Lengua extranjera, es decir, inglés o francés, en los tres cursos y Didáctica de la Lengua Extranjera en al menos un curso. Con el Plan de Estudios de 1971, todo Diplomado debía estar capacitado para impartir la enseñanza globalizada de la $1^{\mathrm{a}}$ etapa de EGB y de la $2^{\mathrm{a}}$ etapa en un área de moderada especialización. Los estudios se distribuían en:

a) Disciplinas comunes, que preparaban para ejercer como profesor generalista en la $1^{\text {a }}$ etapa.

b) De especialización en un área de la EGB, que en nuestro caso se orientaba hacia la especialización moderada en el Área Filológica (Lengua, Literatura Española e Idioma).

16 Alejandro Ávila y Juan A. Holgado Barroso, Formación del Magisterio..., 197.

17 Teresa González Pérez, Enseñando a enseñar, 95. 
c) Optativas, que se proponían profundizar en las especialidades ${ }^{18}$.

Este plan supuso un cambio cualitativo importante por su carácter de estudios universitarios de primer ciclo $^{19}$ en los que se reforzaba las Ciencias de la Educación. Estos estudios resultaban atractivos "como salida profesional en un periodo que se inauguraba con la Ley General de Educación y que ampliaba la escolaridad obligatoria a nivel de EGB hasta los 14 años" 20 . Por tanto, con este plan el problema de las especialidades quedaba resuelto ya que la mayoría de los alumnos había estudiado inglés o francés en el bachillerato, disponían de tres horas semanales durante los tres cursos de la diplomatura y podían cursar asignaturas optativas cuatrimestrales relacionadas con la especialidad ${ }^{21}$, al menos sobre el papel.

Si bien las lenguas extranjeras, especialmente el francés, habían sido parte de la formación de los futuros maestros, no es hasta el Plan de 1971 cuando aparece la especialidad de Filología en los planes de estudios de Magisterio. Los de 1967 y 1971, además del idioma, tienen como novedad la inclusión de la asignatura Didáctica de la lengua extranjera, lo que indica una cierta preocupación no solo porque los futuros maestros dominen la lengua extranjera, sino porque también conozcan los métodos y formas de enseñarla. También se observa cómo a partir de los años 60 la lengua inglesa empieza a ganar terrero frente a la francesa.

El Plan de estudios de 1971 responde a la necesidad de formar maestros capaces de impartir la segunda etapa de la nueva EGB, cuya Ley había sido promulgada en año anterior. Después de la reforma de la LGE muchos maestros se vieron obligados a enseñar lenguas extranjeras sin tener la

18 Daniel Madrid, "Los planes de estudios para la formación inicial de los maestros de inglés”, en Actas de las XII Jornadas Pedagógicas para la enseñanza del inglés, eds. S. Hengge (Granada: GRETA, 1996), 62.

19 Los estudios universitarios en España de la época consistían en las llamadas "diplomaturas" (tres años) y las "licenciaturas" (cinco años). Los tres primeros años de los estudios universitarios se conocían también como "primer ciclo" y los dos restantes como "segundo ciclo".

3020 María Rosa Domínguez Cabrejas, "Perspectiva histórica de los planes de estudio de Magisterio", Revista interuniversitaria de formación del profesorado Núm. 12 (1991): 30.

21 Daniel Madrid, "El maestro especialista en idioma extranjero, una necesidad urgente en la EGB", Apuntes de Educación Núm. 6 (1982): 13. 
preparación adecuada, por lo que tuvieron que realizar cursillos de especialización y perfeccionamiento:

"En el ámbito educativo se contemplaba la lengua inglesa como una materia en creciente expansión y demanda que requería cada vez más la presencia de profesores especializados. Ante su rápida extensión, las medidas más inmediatas hubieron de ser improvisadas en numerosos casos a través de soluciones alternativas como la adaptación de profesorado de otras áreas para la impartición del inglés"22.

\section{La enseñanza de lenguas extranjeras en la Ley General de EDUCACIÓN (1970)}

La LGE, que transformó de la noche a la mañana las antiguas Escuelas Normales en Escuelas Universitarias de Formación del Profesorado de EGB, tenía como objetivos más destacables:

"la generalización de la educación para toda la población; la constitución de un sistema único, obligatorio y gratuito de educación básica que cubriese a la población entre los seis y los catorce años; la búsqueda de unos estándares mínimos de calidad de la enseñanza; el fin del principio de subsidiariedad del Estado reconociéndose su papel en la planificación de la enseñanza y en la provisión de puestos escolares; la reestructuración de todos los niveles educativos"23.

Para Barbero Andrés, "la LGE de 1970 es la medida legislativa que regula, por primera vez de una forma global, la enseñanza obligatoria y generalizada de las lenguas extranjeras en nuestro sistema educativo" 24 . En las Orientaciones Pedagógicas que elaboró el Ministerio de Educación y

22 Javier Barbero Andrés et al. "La enseñanza de la lengua inglesa en España: apuntes para una transición (1970-1995)", Aula Núm. 7 (1995): 136.

23 María Arrazola y José de Hevia, "Evaluación económica de políticas educativas: Una ilustración con la Ley General de la Educación de 1970”, Hacienda Pública Española/ Revista de Economía Pública, Núm. 1 Vol. 164 (2003): 113.

24 Javier Barbero Andrés, "La enseñanza de la lengua inglesa en el sistema educativo español: de la legislación al aula como entidad social (1970-2000)", Cabás Vol. 8 (2012): 74. 
Ciencia en 1970 se exponen los objetivos generales y los específicos del área de lenguaje. Se pretendía la adquisición de un vehículo de comunicación que facilitara:

a) El acercamiento a una segunda cultura, que suponía la adquisición de valores de comprensión y respeto hacia otros pueblos (su forma de vida, de pensar, de reaccionar).

b) Un futuro intercambio comercial, técnico y cultural que evitara frustraciones en los desplazamientos al extranjero (tanto con fines turísticos como profesionales).

c) La adquisición de información, no disponible a través de la lengua materna, para su utilización posterior en la vida profesional ${ }^{25}$.

La LGE asumió algunas de las aportaciones del estructuralismo en la enseñanza de las lenguas. El objetivo fundamental de esta etapa debía ser la adquisición de las cuatro destrezas básicas bajo un enfoque oral, utilizando métodos y técnicas activas. Para favorecer las diversas situaciones de aprendizaje, se proponía una mayor flexibilización de la agrupación tradicional de los alumnos diferenciando entre el trabajo individual, el pequeño grupo, el grupo medio y el gran grupo. Además, se implantó la evaluación continua ${ }^{26}$.

Sin embargo, en la LGE no se tuvieron en cuenta las particularidades metodológicas de enseñar una lengua viva:

"no solo se siguió considerando la lengua extranjera como una asignatura más, prescindiendo de la peculiar naturaleza metodológica con la que se ha de abordar la enseñanza de una lengua viva, sino que el cambio de las preferencias de la mayoría de los alumnos y alumnas hacia el inglés hizo que, una vez más, las autoridades educativas tuvieran que improvisar las medidas con las que cubrir las demandas de una población escolar que comenzaba a recibir clases de idioma extranjero en sexto curso de EGB"27.

27 Javier Barbero Andrés, "La enseñanza de la lengua...", 74. 
Pronto, esa lengua extranjera será el inglés por encima de la lengua francesa:

"En el Estado español, la educación obligatoria se inclina por el inglés, sin que se discuta el necesario equilibrio entre las demandas sociales, la política lingüística europea y el papel educativo de la escuela [...] La administración educativa se deja llevar, a menudo de manera improvisada, por la corriente que impone la enseñanza generalizada del inglés, cediendo así a las presiones sociales de padres e incluso de los propios alumnos que identifican el conocimiento del inglés con el utilitarismo que imponen los tiempos modernos"28.

Y se enseñará no cualquier variedad de la lengua inglesa, sino una muy concreta, el inglés británico y en menor medida el inglés americano:

"Con lo cual se dota a la enseñanza de la lengua inglesa de un sesgo simplista en el cual los valores culturales dominantes son los pertenecientes a los países que, a los ojos de la España expansionista de los setenta, encarnan el éxito histórico en clave exclusivamente económica"29.

\section{El MÉtodo AUdiovisuAL Y AUDIOLINGÜístico}

En el siglo XX surgen dos métodos para la enseñanza de lenguas extranjeras muy parecidos en sus bases teóricas tanto en su concepción de lo que es la lengua, como en sus ideas sobre la forma en que se aprende un idioma. Estos dos métodos fueron: el Oral Approach, también llamado Situational Language Teaching, que surgió y se expandió en Gran Bretaña entre los años 1930 a 1960 y el Audiolingualism que apareció en los EEUU hacia el final de los años $50^{30}$. Cuando el inglés pasa a ser la lengua extranjera

28 Luci Nussbaum, “Aprender a aprender lenguas en la educación primaria”, Signos. Teoría y práctica de la educación Vols. 8/9 (1993): 63.

29 Javier Barbero Andrés, "La enseñanza de la lengua...", 78.

30 Jack S. Richards y Theodore S. Rodgers, Approaches and Methods in Language Teaching (Cambrid- 
más ofertada en España tanto en la enseñanza primaria como secundaria en los años setenta, la mayoría de los libros de texto editados en España seguían los principios de estos métodos de componente estructural.

La concepción de la lengua del Oral Approach y del Audiolingualism era estructuralista. El estructuralismo lingüístico, cuyo iniciador fue Ferdinand de Saussure con su Curso de lingüistica general (1916), considera la lengua como un sistema de interrelaciones y establece los principios de forma y función para delimitar y clasificar las unidades de una lengua. Entre sus principales postulados está la noción del signo lingüístico. El signo lingüístico es la combinación de un concepto o significado y una imagen acústica o significante. El significante es una representación mental de los sonidos que forma un signo mientras que el significado es la representación mental de la realidad, la interpretación del concepto. Los signos no aparecen de forma aislada, sino que se hallan en relación.

"En su concepción y análisis de la realidad, el estructuralismo parte del principio de que el lenguaje, o cualquier otro elemento de la cultura, es una estructura que, a su vez, está constituida por otras subestructuras. Es decir, en el estructuralismo es básico el concepto de unidad formada por componentes vinculados entre sí a modo de entramado de nudos, de relaciones"31.

Saussure nombra dos tipos de relaciones entre los signos lingüísticos: sintagmática (que se da entre dos signos que están presentes en el discurso) y paradigmática (la que se establece entre los signos que están presentes y los que están ausentes). Para Saussure la conexión entre el significado y el significante es arbitraria, es decir, convencional, socialmente construida. No hay ninguna relación intrínseca entre el sonido (significante) y el concepto (significado). Por lo tanto, la conexión entre significante y significado sería

ge: Cambridge University Press, 1986), 31-63; Aquilino Sánchez Pérez, Los métodos en la enseñanza de idiomas. Evolución histórica y análisis didáctico (Madrid: Sociedad General Española de Librería, S.A., 1997), 153-181.

31 Enrique Alcaraz Varó "La lingüística y la metodología didáctica de las lenguas extranjeras", en Enseñanza y aprendizaje de las lenguas modernas, eds. Víctor García Hoz (Madrid: Ediciones Rialp, 1993), 27. 
producto de la interacción humana. La labor de los lingüistas consiste en determinar cuáles son las estructuras de la lengua, desde las más básicas hasta las más complejas para posteriormente producir materiales en los que se insertan estas estructuras, utilizando para ello el vocabulario más frecuente. De ahí la importancia de los listados de frecuencia ${ }^{32}$.

"El estructuralismo partía de una serie de principios que iban a influir directamente en la metodología de las lenguas extranjeras, entre los que se puede destacar la idea de que el lenguaje es fundamentalmente oral; o la afirmación de que una lengua es lo que hablan los nativos de la misma y no lo que digan las autoridades lingüísticas, lo cual subraya el carácter comunicativo del lenguaje"33.

En la elaboración de los libros de texto para la enseñanza de idiomas extranjeros, las teorías estructuralistas se plasmaron en una concepción de lo que hay que enseñar al alumno ordenada en una relación de estructuras gramaticales, un listado de términos y de sonidos que se introducen en cada unidad. De esta forma a una lección determinada le corresponde una estructura gramatical concreta que hay que practicar, una pequeña lista de palabras con las que se debe poner en práctica esa estructura gramatical y dos o tres sonidos nuevos. Las relaciones sintagmática y paradigmática que se establecen entre las palabras se observa en los ejercicios que hace el alumno al sustituir en esa estructura gramatical una determinada palabra por otra.

En cuanto a la teoría del aprendizaje que subyace en ambos métodos, se trata del conductismo. La lengua es un conjunto de hábitos que se adquieren por medio de estímulos condicionados, por lo que el aprendizaje de una lengua consiste en la formación de hábitos que resultan de la repetición continuada. Para el conductismo, el ser humano es un organismo capaz de un amplio repertorio de conductas. La posibilidad de que estas conductas se realicen depende de tres elementos cruciales a la hora de aprender: un estímulo, que sirve para provocar la conducta; una respuesta

32 Aquilino Sánchez Pérez, Los métodos..., 155.

33 Carmen Morales Gálvez et al. La enseñanza de lenguas..., 64-65. 
desencadenada por el estímulo, y un refuerzo, que sirve para señalar si la respuesta es apropiada o inapropiada. Si es apropiada se anima a la repetición de dicha respuesta en el futuro; si es inapropiada se suprime. El refuerzo es un elemento fundamental en el proceso de aprendizaje porque aumenta la probabilidad de que una conducta vuelva a ocurrir y al final se convierta en un hábito ${ }^{34}$.

El conductismo trajo consigo la inclusión de los ejercicios de drill o simulacro en los libros de texto. Estos ejercicios consistían en la repetición por parte del alumno de una estructura gramatical básica varias veces, primeramente de forma coral con el resto de la clase y posteriormente de forma individual. Estas estructuras gramaticales, anteriormente seleccionadas por los lingüistas, estaban ordenadas en función de su complejidad. El objetivo de los ejercicios de drill era crear un hábito en el alumno, por lo que la repetición era fundamental. Por supuesto, la respuesta del alumno debía de ser correcta y entonces se reforzaba ese resultado; si, por el contrario, el alumno cometía un error, el profesor debía corregir ese problema inmediatamente pues de ninguna forma se podía convertir esa respuesta equivocada en un hábito.

En los libros de enseñanza de lengua extranjera se observan diversos tipos de drill. Además de los ejercicios de repetición corales e individuales, también se encuentra el llamado substitution drill que consistía en reemplazar una palabra determinada de la estructura gramatical que se estaba aprendiendo por otra del listado de vocabulario de la lección. Generalmente el vocabulario que se oferta para hacer las sustituciones es básico y cotidiano. En el método británico, también llamado Situational Language Teaching, aprendizaje situacional de la lengua, se tenía en cuenta la situación de habla a la hora de elaborar el listado de términos, si bien esa cuidada elección de vocabulario no siempre fue efectiva y en alguna ocasión la sustitución resultaba poco realista.

En ambos métodos la lengua es primero hablada y luego escrita, por lo que las actividades en el aula favorecían principalmente la expresión oral.

\footnotetext{
34 Jack S. Richards y Theodore S. Rodgers, Approaches and Methods..., 50.
} 
Las actividades son fundamentalmente de memorización de diálogos estructuralmente condicionados y pedagógicamente adaptados y la repetición de estructuras mediante técnicas y procedimientos variados que van desde la repetición simple hasta ejercicios orales más complejos como la reformulación de frases según modelos sugeridos, la reducción de dos frases en una o la expansión de una frase en dos, entre otros ${ }^{35}$. Otros ejercicios eran los dictados y las canciones relacionadas con el tema. Todos estos ejercicios se complementaban con material visual como las flashcards, que eran unas tarjetas con un dibujo o un texto, el magnetófono con sus cintas para practicar la comprensión auditiva y el laboratorio de idiomas. Como el aprendizaje es un proceso inductivo, es decir, a partir de la práctica y de lo concreto, la enseñanza explícita de la gramática no se contempla. Esta debe adquirirse inductivamente, a través de la práctica, y no mediante la reflexión o la explicación del profesor ${ }^{36}$.

Si bien estos métodos tienen mucho en común pues se basan en el estructuralismo y, en cuanto a la teoría del aprendizaje ambos siguen los postulados conductistas, el método audio-lingüístico hace uso de la Lingüística contrastiva, lo que se traduce en el uso de la lengua materna del alumno cuando sea pertinente. En el Oral Approach las clases son siempre en inglés y la traducción está terminantemente prohibida.

\section{Los Libros DE TEXTO PARA LA ENSEÑANZA DE LA LENGUA INGLESA EN LA ENSEÑANZA GENERAL BÁSICA}

Las editoriales españolas pusieron en práctica los principios de estos métodos de base y componente estructural al elaborar los libros de texto para la enseñanza de la lengua inglesa. A mi entender, la serie más representativa de este enfoque metodológico es Ready, Steady, Go de Carmen Echevarría y José Merino, publicada por la editorial Anaya a principio de la década de los setenta del siglo XX. Muy popular, la serie fue reeditada con el título de Inglés $6^{\circ}, 7^{\circ}$ y $8^{\circ}$ y sirvió de base para el curso de inglés del diario español El Sol publicado en 1990.

35 Aquilino Sánchez Pérez, Los métodos..., 162.

36 Aquilino Sánchez Pérez, Los métodos..., 153-181. 
En este apartado analizaremos esa serie de libros de texto para la enseñanza del inglés en los niveles de sexto, séptimo y octavo de EGB. Para ello utilizaremos como guía el formulario de evaluación de materiales educativos diseñado por Jeremy Harmer (1983) y la guía de McDonough y Shaw (2003) si bien unificando algunos criterios con otros para simplificar. El modelo de evaluación de McDonough y Shaw (2003) consta de dos etapas: una evaluación externa e interna. En la primera etapa, el profesor obtiene una idea general del libro ojeando la contraportada, el índice y la introducción. La siguiente etapa, la evaluación interna, es un análisis en profundidad de los materiales. Por su parte, el formulario de evaluación de materiales educativos de Harmer comienza con el profesor dibujando un perfil del alumno y sus necesidades. El formulario de evaluación tiene siete apartados: algunas consideraciones prácticas, la disposición y el diseño gráfico, las actividades, las destrezas, el tipo de lenguaje, el contenido, el material orientativo y las conclusiones.

Esta serie estaba compuesta de los tres libros del alumno, los del profesor que incluían los casetes de los textos orales y unas fichas para la enseñanza individualizada. Los casetes eran fundamentales, pues marcaban el modelo para la pronunciación correcta de las lecciones y fomentaban el acento británico. En cuanto a las fichas, que no se deben confundir con los cuadernos de ejercicios, eran "un tipo de material didáctico en el que, por escrito, se trata de enseñar a aprender al alumno" "37. Se aplicaban tras la lección "plasmando el grado de saber alcanzado en el estudio" 38 . Muy populares en España en esta época, se busca que el estudiante pueda adquirir los contenidos y las formas de trabajo de manera independiente ${ }^{39}$.

"El supremo objetivo de la ficha es crear en el alumno la habilidad mental que necesita para aprender a observar, a buscar, a investigar, a utilizar los medios más idóneos cada vez que quiera

37 Saturnino Arroyo Garrido, El profesor, la programación y la redacción de fichas (Madrid: Editorial Cincel, 1975), 122.

3838 Virgilio Barquero, Métodos activos en la educación general básica (Madrid: Editorial Escuela Española, 1972), 277-8.

39 Alicia Alfaro Valverde y Gilberto Chavarría Chavarría, "La ficha didáctica: una técnica útil y necesaria para individualizar la enseñanza”, Revista Pensamiento Actual Vol. 4 Núm. 5 (2003): 13-23. 
aprender algo sobre un asunto [...] Conviene destacar en principio que en la redacción de fichas han de distinguirse dos tipos fundamentales: fichas en las que se orienta al alumno para que busque la información en otro material didáctico (libros, documentos, etc.), y fichas en las que se le suministra la información completa, sin necesidad de que utilice otro material para informarse" ${ }^{40}$.

En la evaluación externa, un análisis de las portadas originales de los tres libros muestra que estas eran muy coloridas y llamativas, e incluían imágenes relacionadas con la cultura británica. El libro de sexto presentaba una hoja que simulaba un periódico inglés con noticias sobre Hanoi o el viaje del Apolo 16, en cuyo centro se encontraba un dibujo con cuatro de los protagonistas de la serie posando para una fotografía con un beefeater, es decir, uno de los guardias de la Torre de Londres. Además, el número seis está dibujado con la bandera británica. En la portada del libro de séptimo todos los protagonistas infantiles de la serie se refugian en un gigantesco casco de policía, mientras el texto de octavo muestra un paisaje con una torre. En posteriores ediciones de la serie se sustituyeron las imágenes de los protagonistas por un gaitero escocés, la bandera británica, la torre del Big Ben, y un desfile de la guardia real a caballo que continúan el tema de los símbolos de la cultura británica.

Los libros incluyen un índice, bien antes de la primera lección o al final del texto. Contrariamente a lo que sucede con los manuales en la actualidad, en donde cada lección lleva un título, estos índices incluyen información sobre las estructuras gramaticales y el sonido o sonidos que se van a practicar en ese tema. En algunos casos también se especifica el campo semántico que aborda la unidad. Son muy básicos, especialmente si comparamos la información que aportan con la que se encuentra en los manuales actuales que incluye el aspecto de cada destreza (expresión escrita, expresión oral y escrita, comprensión auditiva, comprensión de lectura y audiovisual, interacción oral y escrita) que se va a practicar en esa unidad, la gramática y el vocubulario.

40 Saturnino Arroyo Garrido, El profesor, la programación..., 123. 
Los libros están protagonizados por cuatro niños y cuatro niñas de la edad de los alumnos de los cursos de la Enseñanza General Básica a los que iban dirigidos. Son muy reconocibles, ya que siempre van vestidos de la misma manera, bien con uniforme del colegio, con gafas o un gran sombrero, y representan una característica física. Ellos son los que van introduciendo a los demás personajes.

En la evaluación interna de estos manuales, primeramente se analiza el diseño de las páginas y de cada unidad. En este caso, cada lección consta de seis a ocho páginas cubiertas con grandes dibujos llenos de color y unas pocas palabras debajo de cada imagen. El número de cada unidad tiene como fondo la bandera británica. Este diseño es muy apropiado para los alumnos a los que van dirigidos estos textos, con edades comprendidas entre los once y los catorce años y para los que en la mayoría de los casos era su primera toma de contacto con una lengua extranjera. Los dibujos que se incluyen en estos manuales reflejan muchos de los símbolos de la cultura del Reino Unido tales como el autobús de dos pisos (double decker bus), el policía y el cartero con sus uniformes típicos, el buzón rojo, entre otros.

En cuanto al diseño de cada unidad, generalmente las páginas impares están encabezadas por la palabra "Aprende" mientras que en las pares aparece la palabra "Practica". En las últimas dos hojas, sobre un fondo azul que destaca del resto de la unidad, se incluyen las actividades escritas. Tras aprender y practicar, se incluye un pequeño diálogo con dos recuadros: uno con las palabras nuevas y otro con la pronunciación, es decir, con los sonidos que han sido introducidos en esta unidad. Este formato responde al método audio-lingüístico en el que predomina el uso oral de la lengua antes que el escrito. Un aspecto que destaca es el hecho de que se use el español para explicar los ejercicios o introducir los diferentes apartados de la unidad, pues en el método original la traducción estaba desaconsejada.

En "Aprende" se exponen los nuevos contenidos lingüísticos por medio de ejemplos e ilustraciones. "Practica" son los ejercicios para ensayar y trabajar lo aprendido en el apartado anterior. El diálogo presenta una situación comunicativa con los contenidos aprendidos anteriormente. Tras el diálogo se encuentra un recuadro con los sonidos del inglés que se es- 
tudian en la lección. Los apartados de introducción del tema, diálogo y pronunciación están grabados en las cintas de casete.

Los pasos a seguir en cada unidad son los siguientes: En primer lugar, el alumno escucha y repite la grabación del apartado "Aprende" tres veces. La primera vez, con el libro cerrado; la segunda, mirando las ilustraciones, y la tercera, siguiendo el texto impreso. Estas repeticiones se hacían de forma coral para posteriormente pasar a repetir las frases de forma individualizada. Estas repeticiones son primordiales, pues de lo que se trata es de crear un hábito en el alumno. Si el estudiante dice la frase o la palabra correctamente, el profesor refuerza ese estímulo con una retroalimentación favorable; si se equivoca, el docente tiene que corregir inmediatamente.

Posteriormente, en la sesión "Practica" encontramos oraciones muy similares a las del apartado anterior en las que se ha cambiado un elemento, ya sea gramatical o de vocabulario. En este apartado los alumnos realizan los ejercicios oralmente y/o por escrito. De lo que se trata es de afianzar lo aprendido en la hoja anterior. Algunas lecciones incluyen dictados; se recomienda a los alumnos que se los aprendan de memoria para realizarlos más tarde.

El diálogo es una situación de la vida diaria. Los autores se preocuparon por situar la gramática aprendida en la unidad en un contexto cotidiano. Por ejemplo, si el tema gramatical son las preposiciones de lugar, en el diálogo los protagonistas están en un parque y preguntan dónde están determinados objetos. Si la unidad didáctica es sobre el pasado simple, el diálogo muestra a las protagonistas en el colegio durante el recreo mientras comentan lo que hicieron durante el fin de semana. Como en el primer apartado, el diálogo hay que escucharlo y repetirlo tres veces. Además, se recomienda a los alumnos que aprendan de memoria las intervenciones de uno de los personajes y las digan cuando les toque hablar. En la hoja del diálogo se encuentra un recuadro para practicar la pronunciación y otro con las palabras nuevas. En el ejercicio de pronunciación los alumnos escuchan y repiten varias veces los sonidos o las palabras que se les dan.

Finalmente, en las hojas de actividades escritas se presentan de ocho a 
diez ejercicios escritos en los que el alumno practica principalmente la gramática. En esos ejercicios se transforman oraciones de singular a plural, se convierten frases de forma afirmativa a negativa e interrogativa, se contestan y se formulan preguntas. Como en el resto de la lección, las instrucciones aparecen en español. En algunos casos, especialmente en el libro de sexto, se incorpora alguna actividad oral que consiste en la pronunciación de determinados sonidos, para lo cual se les da el símbolo fonético que se quiere practicar. Por último, tras cada cinco unidades se incluyen dos páginas tituladas "Recuerda" en las que se resumen de forma esquemática el contenido gramatical y en algunas ocasiones el vocabulario de las lecciones precedentes.

En lo que se refiere a los conocimientos socioculturales de la lengua inglesa, los libros incluyen numerosas referencias a la sociedad, los lugares y la cultura británica. Como ya se mencionó, en las ilustraciones de cada unidad se reconocen numerosas imágenes características del Reino Unido como los uniformes de la policía y los carteros, los autobuses de dos pisos o algunos edificios y lugares emblemáticos estrechamente relacionados con la historia del país. Pero, además de estos aspectos más superficiales, los libros incluyen versos típicos ingleses (nursery rbymes) tan conocidos como "Jack and Jill", "Hickory, Dickory, Dock" o "Humpty Dumpty"; canciones infantiles en lengua inglesa muy populares como "Happy Birthday"41, "For he's a jolly good fellow", "My Bonnie lies over the ocean" o "The animals went in two by two", trabalenguas, adivinanzas, algún chiste e incluso los conocidos versos "To be or not to be, that is the question" de la obra de teatro Hamlet de William Shakespeare.

Una particularidad acerca de estos libros es que estaban elaborados por profesores españoles en España. Eso supuso que, a pesar de la gran cantidad de símbolos e imágenes del Reino Unido que aparecen, muchos valores de la España de la época impregnaran los textos. Así, en la unidad 22 de $6^{\circ}$ de EGB cuando se introduce el presente simple para expresar hábitos, los autores con sensatez ilustraron el tema con el horario de uno

41 En la página web de récord Guinness, Guinness World Records, en el año 1998 "Happy Birthday" fue la canción en lengua inglesa más reconocida en todo el mundo, seguida por "For he is a jolly good fellow". 
de los niños protagonistas y las actividades que realiza en cada momento. Cuando llega la noche, Tom cena a las ocho, a las ocho y media ve la televisión y se acuesta a las diez. Ese horario se parece más al de los niños españoles de la época que al de los ingleses.

Otro detalle que actualmente puede llamar la atención es la asociación de una determinada palabra con un sexo indirectamente a través de las ilustraciones. De este modo, al mencionar el tema de las profesiones se usa la palabra nurse (enfermero y enfermera, en español) junto a la imagen de una mujer, mientras que la palabra doctor (doctor y doctora, en español) se asocia a la imagen de un hombre. La madre de algunos de los protagonistas es housewife (ama de casa, en español), si bien no se incluye la palabra para el hombre que hace las tareas de la casa. Este detalle se podría quedar en la simple anécdota si no fuera porque, en mi caso como en el de muchos otros de mi generación, no aprendí que las palabras nurse y doctor se referían a ambos sexos hasta que empecé los estudios universitarios. En los tres años de enseñanza básica y los cuatro de enseñanza secundaria no encontré ningún ejemplo que me dijera lo contrario.

Los tipos de actividades de la serie Ready, Steady, Go responden a los métodos de componente estructuralista que abogan por enseñar a hablar y escuchar antes que a leer y escribir. La unidad empieza con la introducción de la estructura gramatical, si bien en oraciones sueltas debajo de una imagen muy colorida. Esto contrasta con las recomendaciones del Oral Approach que proponía empezar siempre con un diálogo en el que se aplicara la estructura que se va a estudiar en un contexto real. Estas frases se repiten primeramente de forma coral, luego en grupo y por último de forma individual. Para el diálogo grupal se divide la clase en dos. Cada grupo lee o dice de memoria las palabras de uno de los personajes. En las siguientes páginas de la lección, se repasa lo aprendido, bien empleando exactamente los mismos dibujos, bien introduciendo nuevas posibilidades. Estos ejercicios siguen el patrón del substitution drill, es decir, un ejercicio de repetición en el que se sustituye una palabra, pero en vez de un diálogo y un término nuevo para hacer la sustitución, los autores emplean imágenes. Por último, un diálogo breve con la estructura gramatical cierra la unidad. Las actividades escritas del final de cada unidad son introducidas en español y sirven para reforzar las repeticiones de clase, y en unos pocos 
casos un ejercicio oral dedicado a distinguir la pronunciación de determinados sonidos o palabras.

El lenguaje que se emplea en estos libros es básico, muy útil y apropiado para el nivel. Además, se introduce de forma graduada y dentro de un contexto. A diferencia de lo que pasa con los libros actuales que incluyen muchas lecturas sobre temas muy diferentes, la simplicidad de las lecciones favorece el control del vocabulario que debe aprender el alumno. Sin embargo, los diálogos, que estaban diseñados para ser aprendidos de memoria, no resultan naturales ya que fuerzan el uso del vocabulario y la gramática de la unidad. En cuanto a los temas de las unidades, son cotidianos y giran alrededor de los niños protagonistas y sus vidas. Por ejemplo, hay diálogos durante el tiempo del recreo en el colegio, en un parque, las chicas van a comprar a una tienda, los niños hablan sobre sus vacaciones. En cuanto a la inclusión en los libros de texto de algún tipo de guía para el alumno, cada cinco unidades encontramos varias páginas que resumen lo anterior en forma de esquema.

En cuanto al material didáctico complementario que se solía emplear en la clase de lengua inglesa, iba dirigido al estímulo visual y auditivo del alumno e incluía, además del magnetófono y los casetes que ya se han nombrado, el laboratorio de idiomas donde los alumnos en celdas individuales repetían una vez más las estructuras modelo que se habían explicado en clase. Dentro de las ayudas visuales debemos mencionar los carteles, el retroproyector, el proyector de filminas y el de diapositivas, el flanelógrafo, la pizarra, la pizarra magnética, las flashcards, que son, como ya vimos, unas tarjetas con una imagen o un texto: "Pueden contener palabras o números. Su tamaño varía, aunque el más corriente suele ser el de tarjeta postal" En Didáctica de la lengua inglesa en EGB II (1981), editado por el Ministerio de Educación y Ciencia, se recomienda su uso a los maestros pues:

a) Son útiles para los principiantes, a los que se puede pedir que emparejen determinadas palabras con dibujos.

b) O bien, que emparejen grabados con palabras escritas en la pizarra.

42 Didáctica de la Lengua Inglesa en E.G.B. (II) (Madrid: Servicio de Publicaciones del Ministerio de Educación y Ciencia, 1981), 63. 
c) Las flashcards son también útiles como "pista" en los drills orales, especialmente en los de sustitución ${ }^{43}$.

Otros materiales que se recomiendan son un reloj de cartón con agujas movibles, calendarios grandes con los días de la semana y los meses del año, mapas del mundo, postales de distintas ciudades del país cuya lengua se está aprendiendo, teléfonos de juguete para simular conversaciones haciendo de este modo más real la explicación de un diálogo. También aconsejan el uso de películas, directamente relacionadas con los textos y con las estructuras estudiadas, especialmente las que están divididas en episodios $^{44}$. Sin embargo, en la práctica la realidad de los centros educativos parece que fue otra:

"En España el uso del magnetófono en el aula para la presentación de diálogos o de textos no se extendió en la medida que habría sido deseable. Igualmente, a pesar de que también se introdujeron los laboratorios de idiomas en algunos centros, no siempre se hacía uso de ellos por carecer del material adecuado y de personal bien entrenado para su manejo" ${ }^{35}$.

La serie Ready, Steady, Go destaca entre los primeros libros para la enseñanza de la lengua inglesa en la entonces nueva EGB. Los libros respondían a los métodos de componente estructural en boga en la segunda mitad del siglo XX, pero adaptados a la España de la época. Al incluir las cintas de casete con todos los ejercicios y los diálogos grabados, ayudaban a los maestros de esta nueva asignatura con el aspecto oral de la lengua, aspecto que en estos métodos se consideraba fundamental. La lengua de instrucción era generalmente el español y se centraban en la exactitud y la corrección más que en la fluidez; era más importante expresarse correctamente que comunicarse. La repetición oral y posteriormente escrita de unas estructuras, generalmente gramaticales, tenían como objetivo crear un hábito en el alumno y que este posteriormente fuera capaz de aplicar en la calle lo aprendido en el aula. Esta transferencia no era tan sencilla porque en la práctica no se daba la situación lingüística y pragmática exacta

43 Didáctica de la Lengua Inglesa..., 63.

44 Didáctica de la Lengua Inglesa..., 82.

45 Carmen Morales Gálvez et al. La enseñanza de lenguas..., 67. 
en la que aplicar lo aprendido de forma mecánica. Si no se daba el estímulo apropiado, era difícil dar una respuesta.

En las últimas décadas del siglo XX estos métodos de base estructural fueron desechados y sustituidos por estrategias más comunicativas. En su obra Syntactic Structures (1957), el lingüista norteamericano Noam Chomsky cuestiona los fundamentos estructuralistas al afirmar que

"la lengua es algo creativo, innovador y productivo, y que, por tanto, la imitación no tiene ningún sentido ya que las posibilidades de realización en cualquier lengua son infinitas. Chomsky identifica la lengua con la competencia de los hablantes, la cual permite explicitar en infinitas actuaciones o actos productivos la complejidad de mensajes" 46 .

\section{ConClusiones}

Aunque en la actualidad los métodos y los libros de texto de base estructural resultan tediosos y muy poco atractivos para el alumno, en los años 60 y 70 del siglo pasado fueron extremadamente novedosos. Se rompía con la metodología tradicional de gramática y traducción, y se incorporaban ejercicios y materiales innovadores. La prioridad era la lengua oral, es decir, las destrezas de escuchar y hablar el idioma, antes que la lectura y la escritura.

La expansión económica de los 60, ligada al sector turístico, contribuyó al prestigio ascendente de las lenguas modernas y a la inclusión de su docencia en todos los niveles de la enseñanza española. La implantación de la EGB, con la incorporación de la asignatura de Lengua inglesa a partir de sexto curso, trajo consigo la necesidad de un maestro generalista que a su vez tuviese conocimiento en lenguas extranjeras. Las Escuelas Universitarias de Formación del Profesorado de EGB debían preparar a estos nuevos maestros, si bien muchos de los profesores que ejercían su docencia en esas escuelas universitarias habían estudiado en las Facultades de Filosofía y Letras, no eran especialistas en lenguas ni en su didáctica. La noción utilitarista de la importancia de dominar uno o más idiomas empezaba a surgir con fuerza en España.

46 Carmen Morales Gálvez, et al. La enseñanza de lenguas..., 145. 


\section{Bibliografía}

Alcaraz Varó, Enrique. "La lingüística y la metodología didáctica de las lenguas extranjeras". En Enseñanza y aprendizaje de las lenguas modernas, editado por Víctor García Hoz. Madrid: Ediciones Rialp, 1993, 19-108.

Alfaro Valverde, Alicia y Gilberto Chavarría Chavarría. "La ficha didáctica: una técnica útil y necesaria para individualizar la enseñanza". Revista Pensamiento Actual Vol.4 Núm. 5 (2003): 13-23.

Arrazola, María y José de Hevia. "Evaluación económica de políticas educativas: una ilustración con la Ley General de la Educación de 1970”. Hacienda Pública Española / Revista de Economía Pública Vol. 164 Núm. 1 (2003): 111-127.

Arroyo Garrido, Saturnino. El profesor, la programación y la redacción de fichas. Madrid: Editorial Cincel, 1975.

Ávila Fernández, Alejandro y Juan A. Holgado Barroso. Formación del Magisterio en España. La legislación normalista como instrumento de poder y control (1834-2007). Madrid: Ministerio de Educación, Política Social y Deporte. Secretaría General de Educación. Subdirección General de Información y Publicaciones, 2008.

Barbero Andrés, Javier. "La enseñanza de la lengua inglesa en el sistema educativo español: de la legislación al aula como entidad social (1970-2000)". Cabás Vol. 8 (2012): 72-96.

Barbero Andrés, Javier, Gloria Gutiérrez Almarza y Fernando Beltrán Llavador. "La enseñanza de la lengua inglesa en España: apuntes para una transición (1970-1995)”. Aula Vol. 7 (1995): 133-152.

Barquero, Virgilio. Métodos activos en la educación general básica. Madrid: Editorial Escuela Española, 1972.

Didáctica de la Lengua Inglesa (II). Estudios y experiencias educativas. Madrid: Servicio de Publicaciones del Ministerio de Educación y Ciencia, 1981.

Domínguez Cabrejas, María Rosa. "Perspectiva histórica de los planes de estudio de Magisterio". Revista interuniversitaria de formación del profesorado Núm. 12 (1991): 17-32.

European Commission. Education and Training Monitor 2016. https:// ec.europa.eu/education/sites/education/files/monitor2016_en.pdf European Commission. Marco Estratégico: Educación y Formación. http:/ / ec.europa.eu/education/policy/strategic-framework_es 
González Pérez, Teresa. Enseñando a enseñar. Las escuelas de magisterio. Madrid: Mercurio Editorial, 2013.

Harmer, Jeremy. The Practice of English Language Teaching. Londres / Nueva York: Longman, 1983.

McDonough, Jo. y Christopher Shaw. Materials and Methods in ELT. A Teacher's Guide. Malden: Blackwell, 2003.

Madrid, Daniel. "El maestro especialista en idioma extranjero, una necesidad urgente en la EGB”. Apuntes de Educación, Núm. 6 (1982): 12-14.

Madrid, Daniel. "Los planes de estudios para la formación inicial de los maestros de inglés". En Actas de las XII Jornadas Pedagógicas para la enseñanza del inglés, editado por Sergio Hengge. Granada: GRETA, 1996, 62-92.

Madrid, Daniel. "Problemática de la enseñanza de las lenguas extranjeras en España". En La enseñanza de lenguas en el nuevo milenio, editado por Rafael Gómez-Caminero. Granada: Grupo Editorial Universitario, 2001, 11-46.

Morales Gálvez, Carmen, Irene Arrimadas Gómez, Eulalia Ramírez Nueda, Alicia López Gayarre y Laura Ocaña Villuendas. La enseñanza de lenguas extranjeras en España. Madrid: Secretaría General Técnica. Subdirección General de Información y Publicaciones, 2000.

Monterrey, Tomás. "Los estudios ingleses en España (1900-1950): legislación curricular". Atlantis Vol. 25 No. 1 (2003): 63-80.

Nuevas orientaciones para la Educación General Básica. Madrid: Magisterio Español, 1970.

Nussbaum, Luci. "Aprender a aprender lenguas en la educación primaria". Signos. Teoría y práctica de la educación Vols. 8/9 (1995): 62-71.

Richards, Jack S. y Theodore S. Rodgers. Approaches and Methods in Language Teaching. Cambridge: Cambridge University Press, 1986.

Sánchez Pérez, Aquilino. Los métodos en la enseñanza de idiomas. Evolución histórica y análisis didáctico. Madrid: Sociedad General Española de Librería, S.A., 1997.

Para citar este artículo: Vera-Cazorla, María Jesús. "Algunas cuestiones sobre política educativa y la enseñanza de lenguas extranjeras durante el franquismo", Historia Caribe Vol. XIII No. 33 (Julio-Diciembre 2018): 19-46. DOI: http://dx.doi.org/10.15648/hc.33.2018.3 